\title{
Eficacia de la mnemotecnia de la palabra clave en el recuerdo a largo plazo de palabras de un segundo idioma
}

\section{The effectiveness of the keyword mnemonic in long-term retention of words from}

\author{
a second language
}

\author{
Aitziber Goñi-Artola, María Ángeles González \\ Universidade da Coruña
}

\begin{abstract}
Resumen
La mnemotecnia de la palabra clave es una técnica descrita por Atkinson y Raugh en 1975 para el aprendizaje de vocabulario en un segundo idioma. Este trabajo consiste en una revisión sistemática de los resultados publicados, desde entonces hasta la fecha, sobre la eficacia de la mnemotecnia de la palabra clave en el recuerdo a largo plazo de palabras de un segundo idioma. Tras el análisis de las publicaciones localizadas en las diferentes bases de datos consultadas, se constata la ausencia de un resultado uniforme, debido en gran parte a la heterogeneidad de las investigaciones. Todavía son necesarios nuevos estudios para poder llegar a conclusiones definitivas sobre la eficacia de esta técnica en este tipo de recuerdo.

Palabras clave: método de la palabra clave, mnemotecnia, memoria, recuerdo a largo plazo.
\end{abstract}

\begin{abstract}
Keyword mnemonic is a technique for second language vocabulary learning. This technique was first described by Atkinson and Raugh in 1975. This work is a systematic review of the published results, since then up to the date, about the effectiveness of the keyword mnemonic in the long term retention of words from a second language. After the analysis of the publications found in different databases, the absence of an uniform result is verified, probably due to the heterogeneity of the investigations. Further studies are still needed to be able to draw definitive conclusions on the effectiveness of the keyword mnemonic in this type of recall.

Keywords: keyword mnemonic, mnemonics, memory, long-term retention.
\end{abstract}

A la hora de aprender un segundo idioma, el aprendizaje de vocabulario adquiere una importancia fundamental. En 1975, Atkinson y Raugh desarrollaron una técnica basada en la memoria y las imágenes mentales para el aprendizaje de vocabulario de segundos idiomas. Esta técnica, denominada mnemotecnia de la palabra clave, se compone principalmente de dos fases. En la primera fase hay que buscar una palabra en nuestro propio idioma que sea lo más parecida posible a la palabra que queremos aprender, ya sea ese parecido fonético u ortográfico. A esa palabra la denominamos palabra clave. En la segunda fase se forma una imagen mental que relacione esa palabra que se parece (palabra clave) con el significado de la palabra que queremos aprender (Atkinson \& Raugh, 1975).

Se ha investigado la eficacia de la mnemotecnia de la palabra clave (MPC) en diferentes idiomas como el inglés (Ashoori, 2012), español (Wyra, Lawson \& Hungi, 2007), latín (Campos, Pérez-Fabello \& Camino, 2010), turco, japonés, hebreo, galés, italiano, ruso o polaco (Fritz, Morris, Acton, Voelkel \& Etkind, 2007), tagalo (Wang, Thomas \& Oullete, 1992; Wieczynski \& Blick, 1996) u holandés (vanHell \& Mahn, 1997), entre otros.

También se ha investigado la eficacia del método en materiales científicos y matemáticos o de baja frecuencia en el idioma nativo de los sujetos (Brarhler \& Walker, 2008; Brown, 2007; Campos, Rodríguez-Pinal \& Pérez-Fabello, 2013).

Una de las variables que se ha cuestionado desde el principio y que no ha sido fácil de evaluar es la eficacia del método a largo plazo. Con este trabajo se pretende realizar una revisión de los estudios acerca de la eficacia de la MPC a largo plazo cuando se utiliza para el aprendizaje de palabras en un segundo idioma.

\section{Método}

\section{Revisión bibliográfica}

La búsqueda bibliográfica se llevó a cabo en diferentes bases de datos electrónicas. Estas bases de datos fueron: CSIC, Web of Science, PsycInfo, ERIC, SCOPUS y Dialnet. En todas las bases de datos la búsqueda se acotó desde el año 1975 hasta la actualidad (2017).

Los términos utilizados para la búsqueda fueron "keyword mnemonic long term", "mnemotecnia palabra clave largo plazo" y "mnemotecnia palabra clave demorado". Los términos de búsqueda se limitaron al tema o topic. Se seleccionaron artículos publicados en español o en inglés.

\section{Criterios de selección}

Se seleccionaron artículos en los que, al menos uno de los métodos de aprendizaje, fuera la mnemotecnia de la palabra clave, y que el que se recogiera el recuerdo a 
largo plazo. Los artículos seleccionados, siempre que lo permitiera la base de datos, estaban evaluados por expertos.

Se excluyeron todos los trabajos en los que no se utilizara la mnemotecnia de la palabra clave como método de aprendizaje a largo plazo. También se excluyeron los artículos en los que el material a aprender no fueran palabras en un segundo idioma. Los trabajos que no fueran artículos originales de investigación también quedaron fuera de esta selección, incluyendo esto todo tipo de publicaciones secundarias (revisiones, metaanálisis, etc.).

\section{Resultados}

Siguiendo el procedimiento descrito, se obtuvieron un total de 18 artículos diferentes. De la base de datos del CSIC se obtuvieron 3 artículos, de Web of Science 15 artículos, de PsycInfo 7 artículos, de Eric 4 artículos, de SCOPUS 12 artículos, y de Dialnet 2 artículos (véase Figura 1). Hay que tener en cuenta que muchos de los artículos se podrían encontrar en las diferentes bases de datos.

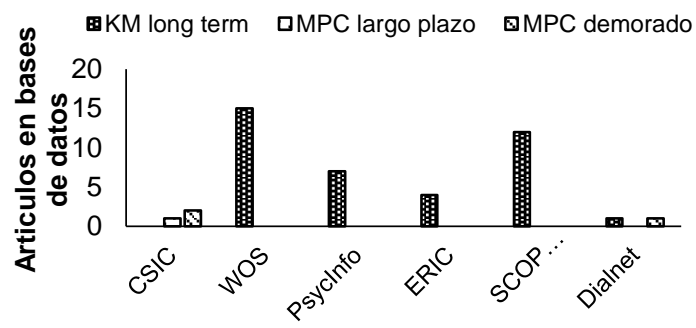

Figura 1. Número de artículos encontrados con los diferentes descriptores en las bases de datos

En este trabajo se ha visto que fue a partir de 1988 cuando se empezó a investigar la efectividad del método

Tabla 1

Tipo de Diseño, Número de Participantes, Número de Palabras e Idioma, Plazo Transcurrido y Principales Resultados de los Trabajos Seleccionados en esta Revisión

\begin{tabular}{llllll}
\hline Autor & Diseño & $\mathrm{N}$ & $\mathrm{N}^{\circ}$ de palabras e idioma & Plazo transcurrido & Resultados principales \\
\hline $\begin{array}{l}\text { Pressley, Levin \& } \\
\text { Ghatala (1988) }\end{array}$ & Experi. & 108 & 24 palabras en latín & Aprox. 2 semanas & $\begin{array}{l}\text { El conocimiento de la estrategia no } \\
\text { influyó en el recuerdo demorado, } \\
\text { aunque si en el uso de la estrategia. } \\
\text { Resultados mejores para los que }\end{array}$ \\
& & & & & $\begin{array}{l}\text { tuvieron la oportunidad de repasar en } \\
\text { la sesión 1. }\end{array}$
\end{tabular}

$\begin{array}{lllll}\begin{array}{l}\text { Wang, Thomas \& } \\ \text { Ouellette (1992) }\end{array} & \text { Experi. } & 79 & \text { 22 palabras en francés } & 1 \text { semana } \\ & \text { Experi. } & 40 & 20 \text { palabras en tagalo } & 1 \text { semana } \\ & \text { Experi. } & 60 & 20 \text { palabras en tagalo } & 1 \text { semana } \\ & \text { Experi. } & 39 & 20 \text { palabras en tagalo } & 1 \text { semana } \\ & & & & \\ & & & & \\ \text { Wang, Thomas, } & \text { Experi. } & 89 & 24 \text { palabras en tagalo } & 2 \text { días } \\ \begin{array}{l}\text { Inzana \& Primicerio } \\ (1993)\end{array} & & & & \\ \text { Wang \& Thomas } & \text { Experi. } & 68 & 24 \text { palabras en inglés } & 2 \text { días }\end{array}$

a largo plazo, aunque el número de artículos publicados al año que aparecen en las bases de datos escogidas nunca son más de dos (Véase Figura 2).

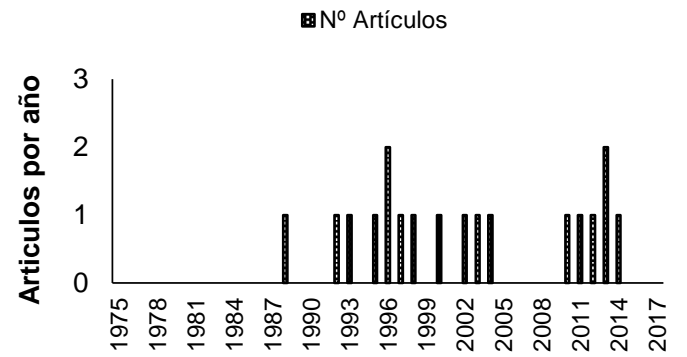

Figura 2. Número de artículos publicados por año

En los 18 artículos encontrados se describían un total de 35 experimentos, debido a que en algunos artículos se incluye más de un experimento. 28 de los experimentos $(80 \%)$ eran de tipo experimental, 2 eran cuasi-experimentales $(5,7 \%)$, en 4 no se especificaba $(11,4 \%)$, y uno de los experimentos $(2,9 \%)$ no cumplía los criterios de inclusión de esta revisión, ya que no incluía recogida de datos a largo plazo. eficacia de la MPC a largo plazo se encuentran en la Tabla 1. Se especifica el tipo de diseño de cada uno de los experimentos, el tamaño de la muestra, el número de palabras utilizadas y el idioma que debían aprender y el plazo transcurrido entre el aprendizaje y la recogida de datos a largo plazo. Los resultados generales de cada estudio figuran también en la misma tabla.
Los principales resultados de los estudios sobre la

Mejor MPC que repetición, pero también un ratio de olvido superior. $\mathrm{El}$ recuerdo demorado fue equivalente entre MPC y repetición.

La MPC fue peor en el recuerdo demorado que método de repetición Repetición recordó más palabras tras 1 semana que MPC.

El recuerdo de MPC decreció más que el de repetición.

Tras dos días no hubo diferencias 
(1995)

$\begin{array}{llll}\text { Experi. } & 48 & 24 \text { palabras en tagalo } & 2 \text { días } \\ \text { Experi. } & 60 & 24 \text { palabras en tagalo } & 2 \text { días }\end{array}$

Thomas \& Wang Experi. $85 \quad 24$ palabras en tagalo 2 días (1996). (Experimento 1 y 2)

No especifi.

24 palabras en tagalo

2 días

Wieczynski,

Experi.

Blick (1996)

vanHell \& Mahn (1997)

(1998)

Experi.

118

24 palabas en tagalo

1 semana

Expe

107

24 palabas en tagalo

5 días

Expe

94

24 palabas en tagalo

2 días y 5 días.

2 días.

Zhang \& Schumm Experi. $60 \quad 20$ palabras en inglés 1 semana (2000)

Campos, Amor \& Experi. González (2002)

Campos, González \& Amor (2003)

Experi.

174 específi.

Campos, Amor \& Experi. González (2004)
30 palabras en latín Experi. $\quad 78 \quad 30$ palabras en latín

No

153

30 palabras en latín

No $\quad 67 \quad 30$ palabras en latín

específi

363

16 palabras en latín

1 semana

1 semana

1 semana

1 semana

1 semana

Expe
$330 \quad 32$ palabras en latín 1 semana entre MPC y contexto semántico.

Contexto semántico obtuvieron mejores resultados que MPC.

Los que utilizaron MPC recordaron más que los de contexto semántico. Cuanto más se practicaba menor era el ratio de olvido en la MPC.

Repetición mejor recuerdo. Mejor los MPC que generaban ellos la Palabra Clave que MPC con PC facilitada por experimentador.

MPC tradicional mejor que MPC con dibujos o repetición.

No hubo diferencias entre MPC y el método de auto-referencia.

Repetición mejor que MPC. Mejor palabras concretas que abstractas.

No diferencias significativas entre los diferentes métodos (MPC y repetición)

MPC mejor que repetición. No diferencias a corto plazo en el grupo que tuvo recuerdo inmediato.

La "práctica de recuperación" no influyó en la MPC y en repetición. Mejor las palabras evaluadas a corto plazo en los que usaron MPC.

Mejor MPC que repetición en palabras evaluadas previamente $\mathrm{y}$ en las "nuevas".

Patrón de olvido comparable en las tres condiciones (repetición, MPC y MPC + interacción). Ejecución de las condiciones de MPC superior.

MPC y un aprendizaje intencional superior a los que aprendieron a través de su propio método.

MPC mejor que repetición, tanto MPC para aprender español como MPC para aprender inglés.

Imágenes bizarras mejor que imágenes normales, independientemente de cómo fueron generadas las palabras clave (por iguales o experimentador).

Repetición mejor que MPC

Repetición mejor que MPC.

Repetición mejor resultado que los que utilizaron MPC reforzada con dibujos.

Repetición mejor resultado que los que utilizaron MPC reforzada con dibujos.

En palabras altas en imagen mejor MPC que repetición. No diferencias cuando la palabra clave era generada por ellos, por el experimentador o por un grupo de iguales.

Mejor cuando las palabras clave las generaba un grupo de iguales que cuando la generaban los sujetos, el 


\begin{tabular}{|c|c|c|c|c|c|}
\hline $\begin{array}{l}\text { Campos, } \\
\text { Pérez-Fabello \& } \\
\text { Camino }(2010)\end{array}$ & $\begin{array}{l}\text { Experi. } \\
\text { Experi. }\end{array}$ & $\begin{array}{l}80 \\
80\end{array}$ & $\begin{array}{l}16 \text { palabras en latín } \\
24 \text { palabras en latín. }\end{array}$ & $\begin{array}{l}1 \text { día } \\
1 \text { día }\end{array}$ & $\begin{array}{l}\text { MPC mejor que repetición. } \\
\text { MPC mejor que repetición. }\end{array}$ \\
\hline $\begin{array}{l}\text { Campos, Camino \& } \\
\text { Pérez-Fabello } \\
(2011)\end{array}$ & $\begin{array}{l}\text { Cuasi-e } \\
\text { xperi. }\end{array}$ & 80 & 16 palabras en latín & 1 día & $\begin{array}{l}\text { Mejor recuerdo con palabras altas en } \\
\text { imagen que con palabras bajas en } \\
\text { imagen. }\end{array}$ \\
\hline $\begin{array}{ll}\text { Saricoban } & \& \\
\text { Basibek (2012) } & \end{array}$ & Experi. & 42 & 20 palabras en inglés & 5 semanas & MPC mejor que método de contexto. \\
\hline $\begin{array}{l}\text { Tavakoli, \& Gerami } \\
\text { (2013) }\end{array}$ & Experi. & 60 & 80 palabras & 2 semana & $\begin{array}{l}\text { Mejor MPC que método pictórico y el } \\
\text { grupo control (no instrucción } \\
\text { específica). }\end{array}$ \\
\hline $\begin{array}{l}\text { Siriganjanavong } \\
\text { (2013) }\end{array}$ & $\begin{array}{l}\text { No } \\
\text { específi }\end{array}$ & 37 & 40 palabras en inglés & 2 semanas & Mejor MPC que método mixto. \\
\hline \multirow[t]{2}{*}{ Dolean (2014) } & Experi. & 101 & 15 palabras en italiano & 1 semana & $\begin{array}{l}\text { Los que utilizaron MPC recordaron } \\
\text { más que el grupo control. }\end{array}$ \\
\hline & $\begin{array}{l}\text { Cuasi-e } \\
\text { xperi. }\end{array}$ & 45 & 30 palabras en italiano & 1 semana y 1 mes. & $\begin{array}{l}\text { No diferencias entre el recuerdo tras } \\
\text { una semana y tras un mes (MPC vs. } \\
\text { método pictórico vs. método verbal). }\end{array}$ \\
\hline
\end{tabular}

\section{Discusión}

En esta revisión, realizada desde 1975 hasta 2017, se observa que es a partir de finales de la década de 1980 cuando comenzaron a publicarse los artículos en los que se investiga la eficacia de la MPC a largo plazo. También se ha constatado que estas publicaciones no son muchas, y que el número de artículos que aparecen por año son pocos o inexistentes. Esto no sorprende si tenemos en cuenta que la MPC es un área temática muy específica dentro de ámbitos como la memoria o el aprendizaje en general. Es interesante destacar que la mayoría de los trabajos encontrados en esta revisión son de tipo experimental, permitiendo así establecer relaciones de causalidad.

Respecto a la eficacia de la MPC a largo plazo en palabras de un segundo idioma, los trabajos analizados muestran resultados variados. Existe un grupo de trabajos en los que la MPC es más eficaz que otros métodos a largo plazo (Carney \& Levin, 1988; Zhang \& Schumm, 2000; Campos et al., 2010; 2011; Saricoban \& Basibek, 2012; Tavakoli \& Gerami, 2013; Siriganjanavong, 2013), pero también existen trabajos donde la eficacia a largo plazo del método se cuestiona (Wang et al., 1992; Wang \& Thomas, 1995; Thomas \& Wang, 1996; Wieczynski \& Blick, 1996; vanHell \& Mahn, 1997; Campos et al., 2003).

En los artículos revisados son diferentes los métodos con los que se compara la MPC. El método con el que más se ha comparado ha sido el método o aprendizaje por repetición. Existen trabajos donde la MPC obtuvo mejores resultados que el método de repetición (Wang et al., 1992; Wang \& Thomas, 1995; Carney \& Levin, 1998; Zhang \& Schumm, 2000; Campos et al., 2004; 2010), y trabajos en los que el método de repetición es superior (Wang et al., 1992; Thomas \& Wang, 1996; experimentador o aprendían por repetición.

MPC mejor que repetición.

Mejor recuerdo con palabras altas en imagen que con palabras bajas en imagen. método pictórico vs. método verbal).
vanHell \& Mahn, 1997; Campos et al., 2003). Wang et al. (1992) observaron que cuando el número de exposiciones a las palabras aumentaba, la ejecución del grupo de repetición era mejor que el de la MPC. También se encontró que la MPC tiene una ratio de olvido superior al método de repetición (Wang et al., 1992; Wang et al, 1993).

También se ha comparado la eficacia de la MPC con el método del contexto, siendo el recuerdo de los que aprendían a través de la MPC, superior (Saricoban \& Basibek, 2012). Cuando la MPC se comparó con el método semántico, la ejecución a largo plazo de este último método fue superior, o no se encontraron diferencias significativas (Wang \& Thomas, 1995).

El tiempo transcurrido entre el aprendizaje y la recogida de datos a largo plazo es una variable que cambia en los diferentes trabajos. Existen trabajos donde la recogida de datos a largo plazo se realizó al día siguiente (Campos et al., 2010), a los dos días (Wang et al. 1993; Wang \& Thomas, 1995; Thomas \& Wang, 1996; Carney, \& Levin, 1998), tras una semana (Wang et al., 1992; Wieczynski \& Blick 1996; Carney \& Levin, 1998; Zhang \& Schumm, 2000; Campos et al., 2002; 2003; 2004) y hasta un mes después (Dolean, 2014). También hay trabajos donde se han realizado dos medidas a largo plazo (vanHell \& Mahn, 1997; Carney \& Levin, 1998; Dolean, 2014).

Los diferentes métodos con los que se compara la MPC, los diversos tiempos transcurridos hasta la recogida de las medidas a largo plazo, pero también el número de palabras empleadas y otros aspectos que cambian de unas investigaciones a otras, pueden ser factores que contribuyen a explicar la diversidad de resultados sobre la eficacia de la MPC que hemos visto en esta revisión. En la mayoría de los experimentos los participantes han sido alumnos, entre otros motivos por 
la fácil accesibilidad que esto permite. Habría que realizar más investigaciones con otros grupos de edad y en otro tipo de contexto. Parece evidente la necesidad de seguir investigando en la eficacia de este método respecto a otros, $\mathrm{y}$ en las variables que pueden afectar a esa eficacia.

\section{Referencias}

Ashoori, A. (2012). Recall of foreign-language vocabulary: effects of keyword, context and word list instructional strategies on long-term vocabulary recall of EFL learners. Journal of Theory and Practice in Education, 8, 54-71.

Atkinson, R. C. \& Raugh, M. R. (1975). An application of the mnemonic keyword method to the acquisition of a Russian vocabulary. Journal of experimental psychology: Human learning and memory, 1, 126-133. http://dx.doi.org/10.1037/0278-7393.1.2.126.

Brahler, J. C. \& Walker, D. (2008). Learning scientific and medical terminology with a mnemonic strategy using an illogical association technique. Advances in Physiology Education, 32, 219-224. http://dx.doi.org/10.1152/advan.00083.2007.

Brown, G. D. (2007). Mathematics vocabulary instruction for current non-proficient students with and without IEPs: A study of three methods of instruction. Dissertation Abstracts International, 69.

Campos, A., Amor, Á. \& González, M. Á. (2002). Presentation of keywords by means of interactive drawings. The Spanish journal of psychology, 5, 102-109.

http://dx.doi.org/10.1017/S1138741600005874.

Campos, A., Amor, A. \& González, M. A. (2004). The importance of the keyword-generation method in keyword mnemonics. Experimental Psychology, 51, 125-131.

http://dx.doi.org/10.1027/1618-3169.51.2.125.

Campos, A., Camino, E. \& Perez-Fabello, M. J. (2011). Using the keyword mnemonics method among adult learners. Educational Gerontology, 37, 327-335. http://dx.doi.org/10.1080/03601271003608886.

Campos, A., González, M. A. \& Amor, A. (2003). Limitations of the mnemonic-keyword method. The Journal of general psychology, 130, 399-413. http://dx.doi.org/10.1080/00221300309601166.

Campos, A., Pérez-Fabello, M. J. \& Camino, E. (2010). Eficacia de la mnemotecnia de la palabra clave en personas adultas. Psicothema, 22, 752-757.

Campos, A., Rodríguez-Pinal, M. D. \& Pérez-Fabello, M. J. (2013). Aprendizaje del idioma gallego mediante la mnemotecnia de la palabra clave, en personas bilingües, altas y bajas en control de imagen. Revista de Investigación en Educación, 11, 50-59.

Carney, R. N. \& Levin, J. R. (1998). Do mnemonic memories fade as time goes by? Here's looking anew! Contemporary Educational Psychology, 23, 276-297. http://dx.doi.org/10.1006/ceps.1998.0970.

Dolean, D. D. (2014). Using the keyword method in the classroom: Is the interacting imagery necessary? System, 45, 17-26.

http://dx.doi.org/10.1016/j.system.2014.04.003.
Fritz, C. O., Morris, P. E., Acton, M., Voelkel, A. R. \& Etkind, R. (2007). Comparing and combining retrieval practice and the keyword mnemonic for foreign vocabulary learning. Applied Cognitive Psychology, 21, 499-526. http://dx.doi.org/10.1002/acp.1287.

Pressley, M., Levin, J. R. \& Ghatala, E. S. (1988). Strategy-comparison opportunities promote long-term strategy use. Contemporary Educational Psychology, 13 , 157-168. http://dx.doi.org/10.1016/0361-476X(88)90016-1.

Saricoban, A. \& Basibek, N. (2012). Mnemonics technique versus context method in teaching vocabulary at upper-intermediate level. Egitim ve Bilim, 37, 251-266.

Siriganjanavong, V. (2013). The Mnemonic Keyword Method: Effects on the Vocabulary Acquisition and Retention. English Language Teaching, 6, 1-10. http://dx.doi.org/10.5539/elt.v6n10p1.

Tavakoli, M. \& Gerami, E. (2013). The effect of keyword and pictorial methods on EFL learners' vocabulary learning and retention. Porta Linguarum, 19, 299-316.

Thomas, M. H. \& Wang, A. Y. (1996). Learning by the Keyword Mnemonic. Journal of Experimental Psychology: Applied, 2, 330-342.

vanHell, J. G. \& Mahn, A. C. (1997). Keyword mnemonics versus rote rehearsal: Learning concrete and abstract foreign words by experienced and inexperienced learners. Language Learning, 47, 507-546. http://dx.doi.org/10.1111/0023-8333.00018.

Wang, A. Y. \& Thomas, M. H. (1995). Effect of Keywords on Long-Term Retention: Help or Hindrance? Journal of Educational Psychology, 87, 468-75.

http://dx.doi.org/10.1037/0022-0663.87.3.468.

Wang, A., Thomas, M., Inzana, C. \& Primicerio, L. (1993). Long-term retention under conditions of intentional learning and the keyword mnemonic. Bulletin of the Psychonomic Society, 31, 545-547. http://dx.doi.org/10.3758/BF03337348.

Wang, A. Y., Thomas, M. H. \& Ouellette, J. A. (1992). Keyword mnemonic and retention of second-language vocabulary words. Journal of Educational Psychology, 84 , 520-528. http://dx.doi.org/10.1037/0022-0663.84.4.520.

Wieczynski, D. M. \& Blick, K. A. (1996). Self-referencing versus the keyword method in learning vocabulary words. Psychological reports, 79, 1391-1394. http://dx.doi.org/10.2466/pr0.1996.79.3f.1391.

Wyra, M., Lawson, M. J. \& Hungi, N. (2007). The mnemonic keyword method: The effects of bidirectional retrieval training and of ability to image on foreign languag evocabulary recall. Learning and Instruction, $\quad 17, \quad 360-371$. http://dx.doi.org/10.1016/j.learninstruc.2007.02.008.

Zhang, Z. \& Schumm, J. S. (2000). Exploring effects of the keyword method on limited English proficient students' vocabulary recall and comprehension. Reading Research and Instruction, 39, 202-221. http://dx.doi.org/10.1080/19388070009558322. 